http://dx.doi.org/10.4314/ajtcam.v11i6.12

\title{
A REVIEW OF THE PHYTOCHEMISTRY, BOTANY, PHARMACOLOGY AND TOXICOLOGY OF ARCTOTIS ARCTOTOIDES (L.F.) O. HOFFM. (ASTERACEAE)
}

\section{Donald Scott Grierson, Wilfred Mbeng Otang and Anthony Jide Afolayan}

\author{
Medicinal Plants and Economic Development Research Center, Botany Department, University of Fort Hare, Private Bag \\ X1314, Alice 5700, Eastern Cape, South Africa. \\ *E-mail: dgrierson@ufh.ac.za; wilfredotang5@yahoo.com; aafolayan@ufh.ac.za
}

\begin{abstract}
Background: Arctotis arctotoides (Asteraceae) is commonly used by the rural people of Eastern Cape for the treatment of epilepsy, indigestion, catarrh and stomach ache. The leaf paste or juice is applied topically in the treatment of wounds and skin disorders. Unfortunately, no previous reviews are available for this important medicinal plant. Hence, the aim of this review is to provide a comprehensive overview of the botany, phytochemistry, pharmacology and toxicology of Arctotis arctotoides.

Methodology: This review was carried out using a comprehensive and systematic literature search on the following databases: Google Scholar, PubMed, Science Direct and Scopus. Searches were undertaken using the key word "Arctotis arctotoides" and the six synonyms of Arctotis arctotoides identified in the Plant List.

Results: In the first phyto-chemical study of Arctotis arctotoides, the authors reported that sesquiterpenoids presence was predominant in the root oil whereas, the essential oils of the leaves, flowers and stems had both monoterpenoids and sesquiterpenoids. The literature survey revealed that Arctotis arctotoides has been investigated in four pharmacological areas, including anti-bacterial, anti-fungal, anti-cancer and anti-oxidant activities. Three toxicity screens for the crude extracts of $A$. arctotoides on cell lines, rats and brine shrimp were identified in the literature.

Conclusion: Detailed studies on the bioactivity of the crude extracts and the isolated phyto-chemicals have provided partial evidence as regards the traditional use of $A$. arctotoides in the treatment of wounds in Eastern Cape of South Africa. However, in order to fully exploit the medicinal potential of $A$. arctotoides, the expansion of existing traditional knowledge into neighboring communities where the plant is not currently in use for the stated indications will support a greater use of the plant in primary healthcare.
\end{abstract}

Key words: Arctotis arctotoides, botany, phyto-chemistry, pharmacology and toxicology

List of abbreviations: ABTS: 2,2'-azino-bis(3-ethylbenzothiazoline-6-sulphonic acid; BHT: Butylated hydroxytoluene;_DPPH: 1,1-diphenyl-2picrylhydrazyl ; FRAP: Ferric reducing ability of plasma; MIC: Minimum inhibitory concentration; MTT: 3-(4,5-dimethythiazol-. 2-yl)-2,5-diphenyl tetrazolium bromide; RPMI 1640 medium: Roswell Park Memorial Institute medium; SEM: Scanning electron microscope; TLC: Thin layer chromatography.

\section{Introduction}

Arctotis arctotoides is a fast-growing, soft, herbaceous plant that is widespread in Eastern Cape Province of South Africa and Lesotho, usually in disturbed areas like road verges. The plant is used by the rural people of Eastern Cape for the treatment of epilepsy, indigestion, catarrh and stomach ache, with the leaf juice or a paste of the leaf applied topically to treat wounds (van der Walt, 2002). To the best of our knowledge, no previous reviews are available regarding the botany, phytochemistry and pharmacological studies of Arctotis arctotoides. Hence, the aim of this review is to provide a comprehensive overview of the botany, phytochemistry, pharmacology and toxicology of Arctotis arctotoides. Additionally, evidence of traditional uses of this important medicinal plant has been examined and recommendations pertaining to future research areas for the plant highlighted.

\section{Methodology}

This review was carried out using a comprehensive and systematic literature search on the medicinal plant Arctotis arctotoides (L.f.) O.Hoffm. The following databases were used: Google-Scholar (http://scholar.google.com), PubMed (http:www.ncbi.nlm.nih.gov/pubmed), ScienceDirect (http://www.sciencedirect.com and Scopus (http://www.scopus.com). Additional search were also undertaken using the six synonyms identified by the plant list (www. the plantlist.org/): Arctotis decurrens; Arctotis micrantha; Arctotis spathuligera; Venidium arctotoides; Venidium decurrens; Venidium spathuligerum

\section{Taxonomy, vernacular names and distribution}

Asteraceae or Compositae (commonly referred to as the aster, daisy, or sunflower family) is an exceedingly large and widespread family of Angiosperms (Jeffrey, 2007). This is the largest family of the flowering plants with more than 24000 - 30000 species and 1600 - 1700 genera (Funk et. al, 2005) worldwide and inhabit almost every environment and continent except Antarctica. The main feature of the family is the composite flower type surrounded by involucral bracts (Bremer, 1994). The genus Arctotis comprises an estimated 50-60 species and belongs to the tribe Arctotideae. The representatives of this genus are indigenous to the Flora of Southern Africa region, with the highest concentration of taxa in Western Cape (Mckenzie, 2006). Arctotis venusta Norl., has the widest distribution, extending to Angola and Southern Zimbabwe, Arctotis stoechadifolia P.J. Bergius is in cultivation and has been introduced into other countries (Pope 1992), while Arctotis arctotoides is widespread throughout the summer / 
rainfall areas of South Africa and Lesotho, usually in disturbed areas like road verges. A. arctotoides is commonly called "botterblom" in Afrikaans, "putswa-pududu" in South Sotho, and "ubushwa" in Xhosa (van der Walt, 2002). The following names are considered as synonyms to A. arctotoides by the plant list (www.plantlist.org): Arctotis decurrens (Less.) O. Hoffm., Arctotis micrantha Thunb. ex DC., Arctotis spathuligera (DC.) O. Hoffm., Osteospermum arctotoides L.f., Venidium arctotoides (L.f.) Less., Venidium decurrens Less., and Venidium spathuligerum DC.

\section{Botany and Traditional Uses}

Arctotis arctotoides is a fast-growing, soft, herbaceous plant with green foliage and butter-yellow daisy flowers almost throughout the year. Each leaf is about $10-15 \mathrm{~cm}$ long with a wavy edge and prominent midrib. The stems are about $20 \mathrm{~cm}$ long and both the leaves and stems are covered with small white hairs. The single daisy flowers which are borne on the stems are about $4 \mathrm{~cm}$ in diameter and contain bright yellow ray florets at the centre. The undersides of the petals are purplish brown and are clearly visible when the flowers are in bud or closed during a cloudy day.

The rural people of Eastern Cape Province of South Africa are known to use Arctotis arctotoides for the treatment of epilepsy, indigestion and catarrh of the stomach and the leaf juice or paste of the leaf is applied topically to treat wounds and fungal infections of the skin (Afolayan, 2003; Otang et al., 2012a).

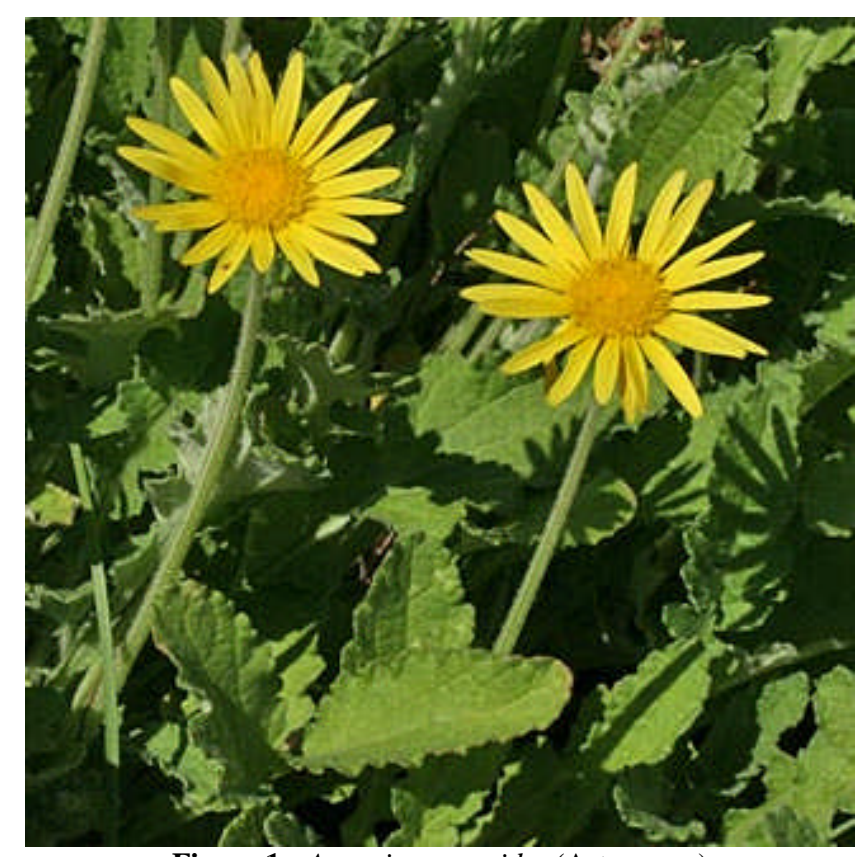

Figure 1: Arctotis arctotoides (Asteraceae)

(Source: http://www.biodiversityexplorer.org/plants/asteraceae/arctotis_arctotoides.htm)

\section{Chemical constituents}

Some of the key phytochemicals that have been isolated and characterized from Arctotis arctotoides and published in academic journals are presented in Table 1. The phytochemicals are labeled 1-33 and include isolated compounds: 27 terpenes, 1 sterol and 2 flavones and families of compounds (flavonoids, poroanthocyanidins and polyphenols) as well. The first phytochemical study on the essential oil of Arctotis arctotoides was carried out by hydro-distillation of the leaves, flowers, stems and roots (Oyedeji et al., 2005). Sesquiterpenoids predominated in the root oil (97.9\%) while the essential oils of the leaves, flowers and stems had both monoterpenoids (34.1-54.5\%) and sesquiterpenoids (38.7-51.9\%). Terpinen-4-ol (5.6-12.6\%), $\beta$-caryophyllene $(6.1-6.9 \%)$ and $\gamma$-curcumene $(6.9-8.49 \%)$ were the major compounds (numbered $1-30)$ common to the three oils (Oyedeji et al., 2005).

Sultana and Afolayan (2007) reported the isolation and identification of a new daucosterol derivative 3-O-[ $\beta$-D-(6'-nadeanoate) glucopyranosyl]- $\beta$-sitosterol and seven known compounds namely: serratagenic acid, stigmasterol, daucosterol, zaluzanin $\mathrm{D}$, dehydrocostuslactone, nepetin, and pedalitin (Figure 1). Subsequently, bioassay guided fractionation of different extracts of Arctotis arctotoides, combined with NMR spectral analysis led to the isolation of glycerol-1-docosanoate, zaluzanin C and perydiscolic acid (Sultana et al., 2008).

\section{Pharmacological Reports}

The literature survey revealed that Arctotis arctotoides has been investigated in four pharmacological areas, including antibacterial, antifungal, anticancer and anti-oxidant activities. The available pharmacological studies with detailed conditions are listed in Table 2. 
http://dx.doi.org/10.4314/ajtcam.v11i6.12

\section{Antibacterial Activities}

A number of in vitro studies have been reported regarding the antibacterial activity of the aqueous, ethanol, methanol and acetone extracts of Arctotis arctotoides (Afolayan, 2003; Afolayan et al., 2007; Sultana and Afolayan, 2007 and Sultana et al., 2008). Afolayan (2003) reported strong antibacterial activity of the acetone, methanol and water whole shoot extracts of Arctotis arctotoides against gram positive bacteria: Bacillus cereus, Bacillus pumilus, Bacillus subtilis, Micrococcus kristinae and Staphylococcus aureus. None of the extracts inhibited Klebsiella pneumoniae and Pseudomonous aeruginosa, both Gram negative bacteria. Afolayan et al. (2007) reported strong antibacterial activity by the acetone extract of Arctotis arctotoides root against Bacillus cereus, Staphylococcus aureus, Micrococcus kristinae, and Streptococcus pyrogens. No MICs were determined and owing to a lack of positive control, the antibacterial activity of Arctotis arctotoides extracts cannot be compared objectively with modern antibiotics, making it difficult to draw conclusion from this study as regards the utilization of the plant for the management of bacterial infections in traditional medicine.

Table 1: Key phytochemicals isolated from Arctotis arctotoides

\begin{tabular}{|c|c|c|c|}
\hline No & Phytochemical & Plant part & Reference \\
\hline & Terpenes & & \\
\hline 1 & $\alpha$-Phellandrene & Leaf, stem & \\
\hline 2 & $\delta$-3-Carene & Leaf & \\
\hline 3 & $\alpha$-Terpinene & Flower, stem & \\
\hline 4 & $p$-cymene & Leaf, stem & \\
\hline 5 & Limonene & Leaf, flower, stem & \\
\hline 6 & 1,8-Cineole & Leaf, flower & \\
\hline 7 & $\gamma$-Terpinene & Leaf, flower, stem & \\
\hline 8 & trans-Sabinene hydrate & Leaf, flower, stem & \\
\hline 9 & Terpinolene & Leaf, flower, stem & \\
\hline 10 & Linalool & Leaf, flower & \\
\hline 11 & $c i s$-Sabinene hydrate & Stem & \\
\hline 12 & cis-p-Menth-2-en-1-ol & Leaf, flower, stem & \\
\hline 13 & trans-p-Menth-2-en-1-ol & Leaf & 1-23: Oyedeji et al., 2005 \\
\hline 14 & Verbenol & Flower & \\
\hline 15 & $p$-Mentha-1,5-dien-8-ol & Flower, stem & \\
\hline 16 & Terpinen-1-ol & Flower & \\
\hline 17 & Terpinen-4-ol & Leaf, flower & \\
\hline 18 & Cymen-8-ol & Flower & \\
\hline 19 & $\alpha$-Terpineol & Leaf, flower, stem & \\
\hline 20 & Myrtenol & Leaf, flower, stem & \\
\hline 21 & trans-Pipertiol & Leaf, flower, stem & \\
\hline 22 & Piperitone & Leaf, flower, stem & \\
\hline 23 & Perlialdehyde & Leaf & \\
\hline 24 & Zaluzanin & Shoot & $\begin{array}{l}\text { Sultana and Afolayan, 2007; } \\
\text { Sultana et al., } 2008\end{array}$ \\
\hline 25 & Perydiscolic acid & Shoot & Sultana et al., 2008 \\
\hline 26 & Serratagenic acid & Shoot & Sultana and Afolayan, 2007 \\
\hline 27 & $\begin{array}{l}\text { Dehydrocostuslactone } \\
\text { Sterol }\end{array}$ & Shoot & Sultana and Afolayan, 2007 \\
\hline 28 & $\begin{array}{l}\text { 3-O-[_ } \beta \text {-D-( } 60 \text { - } \\
\text { nonadeanoate)glucopyranosyl]- } \beta \text {-sitosterol } \\
\text { Flavone }\end{array}$ & Shoot & Sultana and Afolayan, 2007 \\
\hline 29 & Nepetin & Shoot & Sultana and Afolayan, 2007 \\
\hline 30 & Pedalitin & Shoot & Sultana and Afolayan, 2007 \\
\hline 31 & Flavonoids & Roots & Afolayan et al., 2007 \\
\hline 32 & Polyphenol & Roots & Afolayan et al., 2007 \\
\hline 33 & Proanthocyanidins & Roots & Afolayan et al., 2007 \\
\hline
\end{tabular}




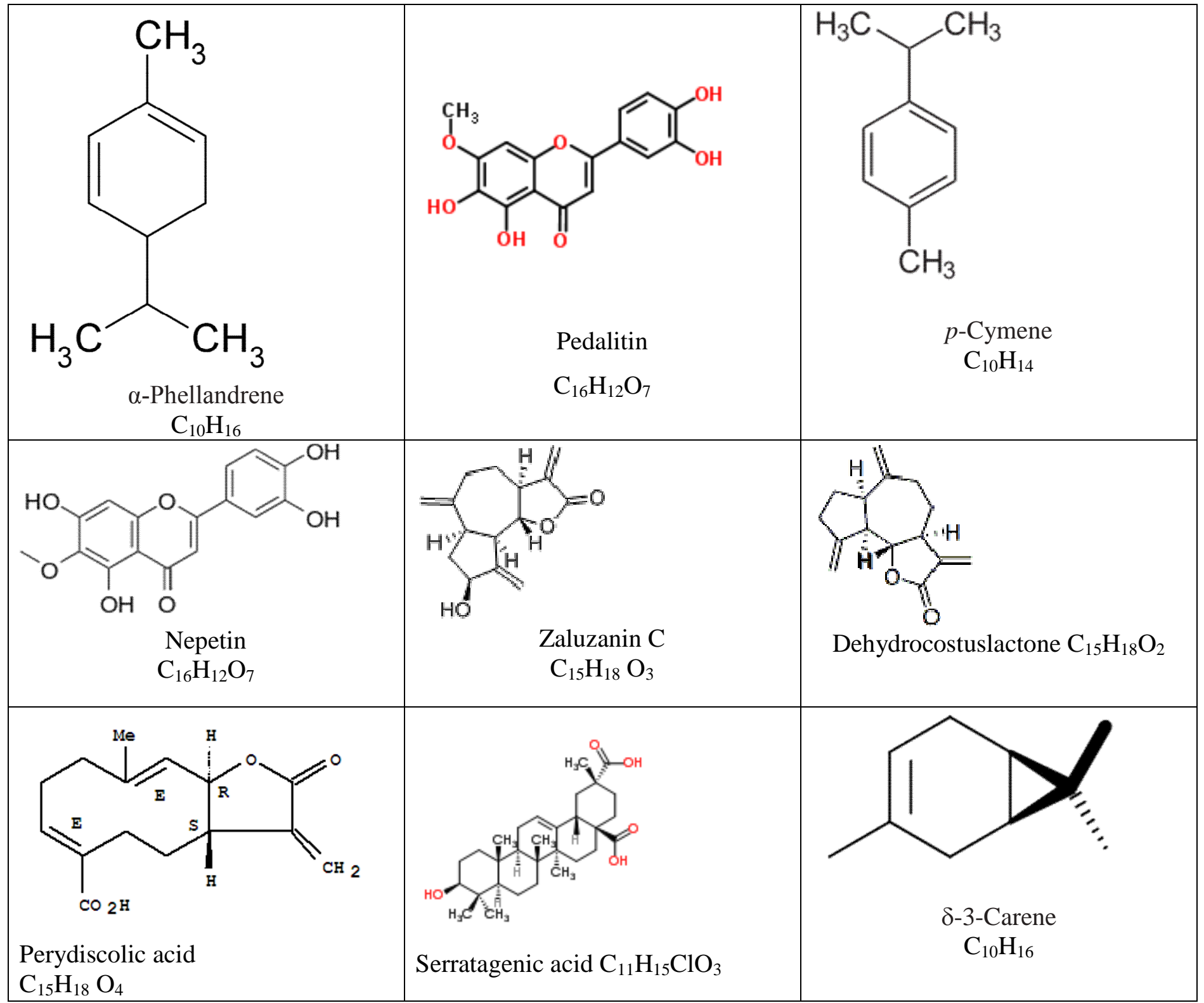

Figure 1: Chemical structures and molecular formulae of some compounds isolated from A. arctotoides (Sultana and Afolayan, 2007; Sultana et al., 2008).

Bio-autographic assay was performed on TLC plates using two Gram positive bacteria: Bacillus subtilis and Staphylococcus aureus and two Gram negative: Escherichia coli and Shigella sonnei and streptomycin as the positive control. Among the compounds (nepetin, stigmasterol, daucosterol, dehydrocostuslactone, serratagenic acid, zaluzanin and pedalitin) isolated from the shoot of A. arctotoides, weak antibacterial activity was observed for nepetin against Bacillus subtilis and Staphylococcus aureus with MICs of $4 \mathrm{mg} / \mathrm{ml}$ and $31 \mathrm{mg} / \mathrm{ml}$ respectively. No antibacterial activity was reported for stigmasterol, daucosterol and dehydrocostuslactone while moderate antibacterial activity by serratagenic acid and pedalitin was observed (Sultana et al., 2008).

\section{Antifungal activity}

A series of studies on the antifungal activity of A. arctotoides have been reported (Afolayan, 2003; Afolayan et al., 2007; Otang et al., 2011; Otang et al., 2012b). The water extract of the shoot of A. arctotoides was particularly inhibitory to the growth of Aspergillus tamari and Penicillum digitatum with inhibitory activity ranging from 50.7 to $95.2 \%$ respectively (Afolayan, 2003). Significant antifungal activity by the acetone extract of the root of A. arctotoides was reported (Afolayan et al., 2007) with growth inhibition ranging from 56.23\% on Aspergillus flavus to 100\% on Penicillium notatum and Mucor heamalis at $5.0 \mathrm{mg} / \mathrm{ml}$. The water extract showed the least inhibitory activity. However, the lack of positive controls in the above studies makes it difficult to conclude about the antifungal activity of $A$. arctotoides. 
Grierson et al., Afr J Tradit Complement Altern Med. (2014) 11(6):118-126

http://dx.doi.org/10.4314/ajtcam.v11i6.12

Table 2: Summary of pharmacological and toxicological studies carried out on Arctotis arctotoides

\begin{tabular}{|c|c|c|c|c|c|c|c|}
\hline $\begin{array}{l}\text { Activity } \\
\text { tested }\end{array}$ & Model used & $\begin{array}{l}\text { Plant part } \\
\text { used/Tested material }\end{array}$ & Extract type & Dosage & Control & Results & Reference \\
\hline Antibacterial & $\begin{array}{l}\text { Nutrient agar medium was } \\
\text { mixed with extract and } \\
\text { bacterial suspension. } \\
\text { Species: Bacillus cereus, } \\
\text { B. pumilus, B. subtilis, } \\
\text { Micrococcus kristinae, } \\
\text { Staphylococcus aureus, } \\
\text { Enterobacter cloacae, } \\
\text { Escherichia coli, } \\
\text { Klebsiella pneumonia, } \\
\text { Pseudomomas } \\
\text { aeruginosa, Serratia } \\
\text { marcescens }\end{array}$ & Whole shoot & $\begin{array}{l}\text { Acetone, } \\
\text { methanol and } \\
\text { water }\end{array}$ & $\begin{array}{l}0.1-5 \\
\mathrm{mg} / \mathrm{ml}\end{array}$ & $\begin{array}{l}\text { Negative: } \\
\text { nutrient agar }+ \\
2 \% \text { solvent. } \\
\text { No positive } \\
\text { control }\end{array}$ & $\begin{array}{l}\text { Strong antibacterial } \\
\text { activity against gram } \\
\text { positive bacteria: Bacillus } \\
\text { cereus, B. pumilus, B. } \\
\text { subtilis, Micrococcus } \\
\text { kristinae and } \\
\text { Staphylococcus aureus. No } \\
\text { MICs determined. None of } \\
\text { the extracts inhibited } \\
\text { Klebsiella } \\
\text { pneumoniae and } \\
\text { Pseudomonous } \\
\text { aeruginosa, both Gram } \\
\text { negative bacteria }\end{array}$ & $\begin{array}{l}\text { Afolayan, } \\
2003\end{array}$ \\
\hline Antibacterial & $\begin{array}{l}\text { Nutrient agar medium was } \\
\text { mixed with extract and } \\
\text { bacterial suspension. } \\
\text { Species: Bacillus cereus, } \\
\text { Staphylococcus } \\
\text { epidermidis, } \\
\text { Staphylococcus aureus, } \\
\text { Micrococcus kristinae, } \\
\text { Streptococcus pyrogens, } \\
\text { Escherichia coli, } \\
\text { Salmonella pooni, } \\
\text { Serratia marcescens, } \\
\text { Pseudomonas aeruginosa, } \\
\text { Klebsiella pneumonia, }\end{array}$ & Root & $\begin{array}{l}\text { Acetone, } \\
\text { methanol and } \\
\text { water }\end{array}$ & $\begin{array}{l}0.1-5 \\
\mathrm{mg} / \mathrm{ml}\end{array}$ & $\begin{array}{l}\text { Negative: } 5 \mathrm{ml} \\
\text { acetone or } \\
\text { methanol } \\
\text { Positive: } \\
\text { Streptomycin }\end{array}$ & $\begin{array}{l}\text { Strong antibacterial } \\
\text { activity by the acetone } \\
\text { extract against } \\
\text { Bacillus cereus, } \\
\text { Staphylococcus aureus, } \\
\text { Micrococcus } \\
\text { kristinae, and } \\
\text { Streptococcus pyrogens. }\end{array}$ & $\begin{array}{l}\text { Afolayan } \\
\text { et al., } \\
2007\end{array}$ \\
\hline Antibacterial & $\begin{array}{l}\text { Bioautographic assay was } \\
\text { performed on TLC plates } \\
\text { using two Gram positive } \\
\text { bacteria: Bacillus subtilis } \\
\text { and Staphylococcus } \\
\text { aureus and two Gram } \\
\text { negative: Escherichia coli } \\
\text { and Shigella sonnei }\end{array}$ & $\begin{array}{l}\text { Compounds isolated } \\
\text { from shoot of } A \text {. } \\
\text { arctotoides: nepetin } \\
\text { stigmasterol, } \\
\text { daucosterol, } \\
\text { dehydrocostuslactone. } \\
\text { serratagenic acid, } \\
\text { zaluzanin and pedalitin }\end{array}$ & & $\begin{array}{l}2-250 \\
\mu \mathrm{g} / \mathrm{ml}\end{array}$ & $\begin{array}{l}\text { Negative: } \\
\text { Dimethyl } \\
\text { sulphoxide } \\
\text { Positive: } \\
\text { Streptomycin }\end{array}$ & $\begin{array}{l}\text { Significant antibacterial } \\
\text { activity of nepetin against } \\
\text { Bacillus subtilis and } \\
\text { Staphylococcus aureus } \\
\text { with MICs of } 4 \mathrm{mg} / \mathrm{ml} \text { and } \\
31 \mathrm{mg} / \mathrm{ml} \text { respectively. No } \\
\text { antibacterial activity by } \\
\text { stigmasterol, } \\
\text { daucosterol and } \\
\text { dehydrocostuslactone. } \\
\text { Moderate antibacterial } \\
\text { activity by serratagenic } \\
\text { acid and pedalitin }\end{array}$ & $\begin{array}{l}\text { Sultana } \\
\text { and } \\
\text { Afolayan, } \\
2007\end{array}$ \\
\hline Antibacterial & $\begin{array}{l}\text { Bioautographic assay was } \\
\text { performed on TLC plates } \\
\text { using Bacillus cereus, } B \text {. } \\
\text { subtilis, Staphylococcus } \\
\text { aureus, Staphylococcus } \\
\text { epidermidus, Escherichia } \\
\text { coli, and Shigella sonnei }\end{array}$ & $\begin{array}{l}\text { Compounds isolated } \\
\text { from shoot of } A \text {. } \\
\text { arctotoides: glycerol-1- } \\
\text { docosanoate, zaluzanin } \\
\text { C and perydiscolic acid }\end{array}$ & Ethanol & $\begin{array}{l}\text { Not } \\
\text { mentioned }\end{array}$ & $\begin{array}{l}\text { Negative: } \\
\text { DMSO } \\
\text { Positive: } \\
\text { Streptomycin }\end{array}$ & $\begin{array}{l}\text { Moderate antibacterial } \\
\text { activity of compounds } \\
\text { isolated from } A \text {. } \\
\text { arctotoides against Gram } \\
\text { positive and Gram } \\
\text { negative bacteria }\end{array}$ & $\begin{array}{l}\text { Sultana et } \\
\text { al., } 2008\end{array}$ \\
\hline Antifungal & $\begin{array}{l}\text { Nutrient agar medium was } \\
\text { mixed with extract and } \\
\text { fungal suspension. } \\
\text { Species: Aspergillus } \\
\text { flavus, Aspergillus } \\
\text { tamarii, Cladosporium } \\
\text { herbarum, Cladosporium } \\
\text { sphaerospermum, } \\
\text { Penicillium digitatum, } \\
\text { Penicillium italicum }\end{array}$ & Shoot & $\begin{array}{l}\text { Acetone, water } \\
\text { and methanol }\end{array}$ & $\begin{array}{l}0.1-5 \\
\mathrm{mg} / \mathrm{ml}\end{array}$ & $\begin{array}{l}\text { Negative: } \\
\text { nutrient agar }+ \\
2 \% \text { solvent. } \\
\text { No positive } \\
\text { control }\end{array}$ & $\begin{array}{l}\text { Extracts showed } \\
\text { significant } \\
\text { growth inhibition against } \\
\text { all the fungi tested. The } \\
\text { water extract was } \\
\text { particularly inhibitory to } \\
\text { the growth of Aspergillus } \\
\text { tamari and Penicillum } \\
\text { digitatum with inhibitory } \\
\text { activity ranging from } \\
50.7 \text { to } 95.2 \% \text { respectively }\end{array}$ & $\begin{array}{l}\text { Afolayan, } \\
2003\end{array}$ \\
\hline Antifungal & $\begin{array}{l}\text { Prepared potato dextrose } \\
\text { agar plates containing } A \text {. } \\
\text { arctotoides extract at } \\
\text { concentrations of } 5.0,1.0 \text {, } \\
0.5 \text { and } 0.1 \mathrm{mg} / \mathrm{ml} \text {, were } \\
\text { inoculated with plugs } \\
\text { obtained from the actively } \\
\text { growing margin of the }\end{array}$ & Root & $\begin{array}{l}\text { Acetone, water } \\
\text { and methanol }\end{array}$ & $\begin{array}{l}0.1-5 \\
\mathrm{mg} / \mathrm{ml}\end{array}$ & $\begin{array}{l}\text { Negative: } 5 \mathrm{ml} \\
\text { acetone or } \\
\text { methanol } \\
\text { Positive: } \\
\text { Streptomycin }\end{array}$ & $\begin{array}{l}\text { Significant antifungal } \\
\text { activity by the acetone and } \\
\text { methanol extracts with } \\
\text { growth } \\
\text { inhibition ranging from } \\
56.23 \% \text { on Aspergillus } \\
\text { flavus to } \\
100 \% \text { on Penicillium }\end{array}$ & $\begin{array}{l}\text { Afolayan } \\
\text { et al., } \\
2007\end{array}$ \\
\hline
\end{tabular}


Grierson et al., Afr J Tradit Complement Altern Med. (2014) 11(6):118-126

http://dx.doi.org/10.4314/ajtcam.v11i6.12

\begin{tabular}{|c|c|c|c|c|c|c|c|}
\hline & $\begin{array}{l}\text { fungi plates and incubated } \\
\text { at } 25^{\circ} \mathrm{C} \text {. The diameter of } \\
\text { the fungal growth was } \\
\text { measured }\end{array}$ & & & & & $\begin{array}{l}\text { notatum and } \text { Mucor } \\
\text { heamalis at } 5.0 \mathrm{mg} / \mathrm{ml} \text {. The } \\
\text { water } \\
\text { extract showed the least } \\
\text { inhibitory activity }\end{array}$ & \\
\hline Antifungal & $\begin{array}{l}\text { Diffusion method on } \\
\text { sabouraud dextrose agar } \\
\text { plate. Wells were made on } \\
\text { the plate to put the extract, } \\
\text { negative and positive } \\
\text { control. Species: Candida } \\
\text { albicans, Candida krusei, } \\
\text { Candida glabrata, } \\
\text { Cryptococcus } \\
\text { neoformans, Aspergillus } \\
\text { fumigatus, Aspergillus } \\
\text { niger, Trychophyton } \\
\text { tonsurans, Trychophyton } \\
\text { mucoides, Microsporum } \\
\text { gypseum and } \\
\text { Microsporum canis }\end{array}$ & Leaves & $\begin{array}{l}\text { Hexane and } \\
\text { acetone }\end{array}$ & $\begin{array}{l}0.005- \\
5 \mathrm{mg} / \mathrm{ml}\end{array}$ & $\begin{array}{l}\text { Negative: } \\
\text { corresponding } \\
\text { solvent } \\
\text { Positive: } \\
\text { Nystatin, } \\
\text { Amphotericin } \\
\text { B and } \\
\text { Grieseofulvin }\end{array}$ & $\begin{array}{l}\text { The hexane extract was } \\
\text { active against } 6 \text { out of the } \\
10 \text { fungi, the acetone } \\
\text { extract was active against } 7 \\
\text { out } \\
\text { of the } 10 \text { tested fungi } \\
\text { with zones of inhibition } \\
\text { varying from } 8 \text { to } 32 \mathrm{~mm} \text {, } \\
\text { while none of the aqueous } \\
\text { extracts was active against } \\
\text { any of the fungi. Highest } \\
\text { antifungal activity was } \\
\text { obtained with } \\
\text { the hexane extract of } A \text {. } \\
\text { arctotoides with MICs of } \\
0.005 \mathrm{mg} / \mathrm{ml} \text { against } T \text {. } \\
\text { mucoides and } 0.04 \mathrm{mg} / \mathrm{ml} \\
\text { against } A \text {. niger }\end{array}$ & $\begin{array}{l}\text { Otang et } \\
\text { al., } 2012\end{array}$ \\
\hline Antifungal & $\begin{array}{l}\text { Spore suspensions of } \\
\text { Candida albicans, } \\
\text { Candida glabrata, } \\
\text { Aspergillus fumigatus, } \\
\text { and Microsporum canis } \\
\text { were aseptically } \\
\text { transferred into the test } \\
\text { tubes containing various } \\
\text { concentrations of the } \\
\text { acetone extract of } A \text {. } \\
\text { arctotoides dissolved in } \\
\text { sterilised sabouraud } \\
\text { dextrose. The } \\
\text { tubes were incubated at } 37 \\
{ }^{\circ} \text { C. Morphological } \\
\text { alterations of fungal } \\
\text { mycelia were assessed by } \\
\text { scanning electron } \\
\text { microscope }\end{array}$ & Leaves & Acetone & $\begin{array}{l}0.32- \\
5 \mathrm{mg} / \mathrm{ml}\end{array}$ & $\begin{array}{l}\text { Negative: } \\
\text { sabouraud } \\
\text { dextrose broth. } \\
\text { No positive } \\
\text { control }\end{array}$ & $\begin{array}{l}\text { Remarkable morphological } \\
\text { alterations in the fungal } \\
\text { mycelia, loss of turgidity } \\
\text { and uniformity, collapse of } \\
\text { entire hyphae to evident } \\
\text { destruction of the hyphae }\end{array}$ & $\begin{array}{l}\text { Otang et } \\
\text { al., } 2011\end{array}$ \\
\hline Anticancer & $\begin{array}{l}\text { Extracts of } A \text {. arctotoides } \\
\text { were assayed in } 3 \text { cell } \\
\text { lines (renal TK10, breast } \\
\text { MCF7, melanoma } \\
\text { UACC62) in } 96 \text { well } \\
\text { microtitre plates } \\
\text { containing RPMI } 1640 \\
\text { medium and incubated at } \\
37^{\circ} \mathrm{C}, 5 \% \mathrm{CO}_{2} \text {. The assay } \\
\text { results for the extracts } \\
\text { screened were reported as } \\
\text { total growth inhibition } \\
\text { values }\end{array}$ & Whole plant & $\begin{array}{l}\text { Dichloromethane } \\
\text { and methanol }\end{array}$ & $\begin{array}{l}6.25- \\
100 \mathrm{ug} / \mathrm{ml}\end{array}$ & $\begin{array}{l}\text { Negative: } \\
\text { DMSO + } \\
\text { RPMI } \\
\text { Positive: } \\
\text { etoposide }\end{array}$ & $\begin{array}{l}\text { Moderate anticancer } \\
\text { activity with total growth } \\
\text { inhibition of } 12.70,16.81 \\
\text { and } 13.74 \mu \mathrm{g} / \mathrm{ml} \text { in renal } \\
\text { TK10, breast MCF7 and } \\
\text { Melanoma UACC62 cell } \\
\text { lines respectively }\end{array}$ & $\begin{array}{l}\text { Fouche et } \\
\text { al., } 2008\end{array}$ \\
\hline Toxicity & $\begin{array}{l}\text { Male Wistar rats which } \\
\text { had fasted for } 16 \text { hours } \\
\text { were administered with } \\
\text { graded doses of } A \text {. } \\
\text { arctotoides extract, } \\
\text { allowed assess to food and } \\
\text { water for } 48 \text { hours. Serum } \\
\text { and tissue homogenate } \\
\text { (liver, kidney) were used } \\
\text { to estimate } \\
\text { haematological } \\
\text { parameters }\end{array}$ & Shoot & Water & $\begin{array}{l}400-3,200 \\
\mathrm{mg} / \mathrm{kg} \\
\text { body } \\
\text { weight }\end{array}$ & $\begin{array}{l}\text { Negative: } \\
\text { Group of rats } \\
\text { receiving } \\
\text { distilled water }\end{array}$ & $\begin{array}{l}\text { No significant change in } \\
\text { the red blood cell count } \\
\text { and haemoglobin } \\
\text { concentration, no } \\
\text { significance increase in } \\
\text { white blood cell count and } \\
\text { its differentials. Significant } \\
\text { decrease in the levels of } \\
\text { some liver enzymes and } \\
\text { blood urea nitrogen. No } \\
\text { significant lesions were } \\
\text { observed in the organs } \\
\text { examined }\end{array}$ & $\begin{array}{l}\text { Jimoh et } \\
\text { al., } 2008\end{array}$ \\
\hline Toxicity & Toxicity assessment was & Leaf & Hexane and & $0.125-2$ & Negative: Sea & Hatching success was & Otang et \\
\hline
\end{tabular}


Grierson et al., Afr J Tradit Complement Altern Med. (2014) 11(6):118-126

\section{http://dx.doi.org/10.4314/ajtcam.v11i6.12}

\begin{tabular}{|c|c|c|c|c|c|c|c|}
\hline & $\begin{array}{l}\text { based on both the } \\
\text { percentage of hatching of } \\
\text { cysts and lethality of } \\
\text { hatched nauplii in } \\
\text { different concentrations of } \\
\text { plant extracts and controls }\end{array}$ & & acetone & $\mathrm{mg} / \mathrm{ml}$ & $\begin{array}{l}\text { water } \\
\text { Positive: } \\
\text { amphotericin } \\
\text { B }\end{array}$ & $\begin{array}{l}\text { significantly lower in cysts } \\
\text { incubated in the hexane } \\
(5.10 \%) \text { and acetone } \\
(5.20 \%) \text { extracts as } \\
\text { compared to the control. } \\
\text { Both the acetone and the } \\
\text { hexane extract exhibited } \\
\text { significant brine shrimp } \\
\text { lethality with } \mathrm{LC}_{50} \text { values } \\
\text { of } 0.87 \text { and } 0.89 \mathrm{mg} / \mathrm{ml} \\
\text { respectively }\end{array}$ & al., 2013a \\
\hline Toxicity & $\begin{array}{l}\text { In vitro cytotoxicity } \\
\text { evaluation of } A \text {. } \\
\text { arctotoides extracts } \\
\text { against the Chang liver } \\
\text { cell line. Different } \\
\text { concentrations of the } \\
\text { extracts were added into } \\
24 \text {-hour cultured cells and } \\
\text { incubated for } 72 \text { hours, } 37 \\
{ }^{\circ} \mathrm{C}, 5 \% \mathrm{CO}_{2} \text {. Cell survival } \\
\text { was evaluated using the } 3 \text { - } \\
(4,5 \text {-dimethylthiazol-2- } \\
\text { yl)-2, 5- } \\
\text { diphenyltetrazolium } \\
\text { bromide (MTT) assay }\end{array}$ & Leaf & $\begin{array}{l}\text { Hexane and } \\
\text { acetone }\end{array}$ & $0-50 \mathrm{ug} / \mathrm{ml}$ & $\begin{array}{l}\text { Positive: } \\
\text { Griseofulvin }\end{array}$ & $\begin{array}{l}\text { The } \mathrm{LC}_{50} \text { values of the } \\
\text { acetone and hexane } \\
\text { extracts of } A \text {. arctotoides } \\
\text { were } 17.4 \text { and } 12.4 \mu \mathrm{g} / \mathrm{ml} \\
\text { respectively and were not } \\
\text { significantly lower than } \\
\text { that of the positive } \\
\text { griseofulvin }(9.02 \mu \mathrm{g} / \mathrm{ml})\end{array}$ & $\begin{array}{l}\text { Otang et } \\
\text { al., } 2013 \mathrm{~b}\end{array}$ \\
\hline Antioxidant & $\begin{array}{l}\text { In vitro DPPH free-radical } \\
\text { scavenging activity }\end{array}$ & Root & $\begin{array}{l}\text { Acetone, } \\
\text { methanol and } \\
\text { water }\end{array}$ & $\begin{array}{l}0.02- \\
0.1 \mathrm{mg} / \mathrm{ml}\end{array}$ & $\begin{array}{l}\text { Negative: } \\
\text { corresponding } \\
\text { solvent. } \\
\text { Positive: } \\
\text { Ascorbic acid } \\
\text { and BHT }\end{array}$ & $\begin{array}{l}\text { The methanol, water and } \\
\text { acetone extracts showed a } \\
\text { lower potency than } \\
\text { ascorbic acid and BHT at } \\
\text { all tested concentrations. } \% \\
\text { inhibition at } 0.1 \mathrm{mg} / \mathrm{ml} \text { - } \\
\text { methanol: } 68.2 \% \text {, water: } \\
7.5 \% \text {, acetone: } 61.8 \%\end{array}$ & $\begin{array}{l}\text { Afolayan } \\
\text { et al., } \\
2007\end{array}$ \\
\hline Antioxidant & $\begin{array}{l}\text { In vitro ABTS free-radical } \\
\text { scavenging activity }\end{array}$ & Root & $\begin{array}{l}\text { Acetone, } \\
\text { methanol and } \\
\text { water }\end{array}$ & $\begin{array}{l}0.02- \\
0.1 \mathrm{mg} / \mathrm{ml}\end{array}$ & $\begin{array}{l}\text { Negative: } \\
\text { corresponding } \\
\text { solvent. } \\
\text { Positive: BHT }\end{array}$ & $\begin{array}{l}\text { The acetone root extract } \\
\text { showed a higher potency } \\
\text { than BHT at } 0.1 \mathrm{mg} / \mathrm{ml} . \% \\
\text { inhibition. Acetone: } \\
96.7 \% \text {, methanol: } 95.5 \% \text {, } \\
\text { water: } 91.1 \% \text { and BHT: } \\
96.3 \%\end{array}$ & $\begin{array}{l}\text { Afolayan } \\
\text { et al., } \\
2007\end{array}$ \\
\hline Antioxidant & $\begin{array}{l}\text { In vitro ABTS free-radical } \\
\text { scavenging activity }\end{array}$ & Root & $\begin{array}{l}\text { Acetone, } \\
\text { methanol and } \\
\text { water }\end{array}$ & $\begin{array}{l}0.02- \\
0.1 \mathrm{mg} / \mathrm{ml}\end{array}$ & $\begin{array}{l}\text { Negative: } \\
\text { corresponding } \\
\text { solvent } \\
\text { Positive: BHT }\end{array}$ & $\begin{array}{l}\text { The acetone root extract } \\
\text { showed a higher potency } \\
\text { than BHT at } 0.1 \mathrm{mg} / \mathrm{ml} . \% \\
\text { inhibition. Acetone: } \\
96.7 \% \text {, methanol: } 95.5 \% \text {, } \\
\text { water: } 91.1 \% \text { and BHT: } \\
96.3 \%\end{array}$ & $\begin{array}{l}\text { Afolayan } \\
\text { et al., } \\
2007\end{array}$ \\
\hline Antioxidant & $\begin{array}{l}\text { In vitro FRAP assay: } \\
\text { ability of the extracts to } \\
\text { reduce TPRZ-Fe (III) } \\
\text { complex to TPTZ-Fe (II) } \\
\text { was assessed }\end{array}$ & Root & $\begin{array}{l}\text { Acetone, } \\
\text { methanol and } \\
\text { water }\end{array}$ & $\begin{array}{l}0.02- \\
0.1 \mathrm{mg} / \mathrm{ml}\end{array}$ & $\begin{array}{l}\text { Negative: } \\
\text { corresponding } \\
\text { solvent. } \\
\text { Positive: BHT, } \\
\text { ascorbic acid, } \\
\text { catechin }\end{array}$ & $\begin{array}{l}\text { The FRAP values for the } \\
\text { acetone }(80.43) \text { and } \\
\text { methanol }(81.86) \text { extracts } \\
\text { were significantly lower } \\
\text { than those of ascorbic } \\
\text { Acid (1626.5) and catechin } \\
\text { (971.6) but higher than that } \\
\text { of BHT }(62.3)\end{array}$ & $\begin{array}{l}\text { Afolayan } \\
\text { et al., } \\
2007\end{array}$ \\
\hline
\end{tabular}

Otang et al. (2011) investigated the effect of the acetone extract of A. arctotoides on the growth and ultra-structure of some opportunistic fungi (Candida albicans, Candida glabrata, Aspergillus fumigatus, and Microsporum canis) associated with HIV/AIDS by means of scanning electron microscope (SEM). Remarkable morphological alterations in the fungal mycelia which were attributed to the loss of cell wall strength ranged from loss of turgidity and uniformity, collapse of entire hyphae to evident destruction of the hyphae. The inhibition of mycelia growth ranged from $0 \%$ in $A$. fumigatus at a concentration of $0.32 \mathrm{mg} / \mathrm{ml}$ of plant extract to $65.3 \%$ in $C$. albicans at the highest concentration of $5 \mathrm{mg} / \mathrm{ml}$. The plant extract inhibited mycelia growth in $C$. albicans and $M$. canis in a dose-dependent manner. The observed alterations in the fungal ultra-structure were attributed to the impact of the plant extract on the cell wall with the resultant changes in the fungal hyphae. The hexane and the acetone extracts of the leaves of $A$. artotoides were reported to be active against 6 out of 10 fungi, against 7 out of the 10 tested fungi respectively, with zones of inhibition varying from 8 to $32 \mathrm{~mm}$ (Otang et al., 2012). Highest antifungal activity was obtained with the hexane extract of A. arctotoides with MICs of $0.005 \mathrm{mg} / \mathrm{ml}$ against T. mucoides and $0.04 \mathrm{mg} / \mathrm{ml}$ against $A$. niger, and considering the fact that the mean inhibition zone diameters of $A$. arctotoides (hexane) and the positive control (Nystatin) were not significantly different $(P>0.05)$ suggests that $A$. arctotoides appear to have high efficacy with a broad spectrum of antifungal activity 
http://dx.doi.org/10.4314/ajtcam.v11i6.12

\author{
Anticancer activity \\ Dichloromethane and methanol extracts of $A$. arctotoides were assayed in 3 cell lines (renal TK10, breast MCF7, melanoma UACC62) in \\ 96 well micro-titre plates containing RPMI 1640 medium and incubated at $37^{\circ} \mathrm{C}, 5 \% \mathrm{CO}_{2}$ (Fouche et al., 2008). The assay results for the extracts \\ screened were reported as total growth inhibition values. Moderate anticancer activity with total growth inhibition at $12.70,16.81 \mathrm{and} 13.74 \mu \mathrm{g} / \mathrm{ml}$ in \\ renal TK10, breast MCF7 and Melanoma UACC62 cell lines respectively
}

\title{
Antioxidant activity
}

The in vitro antioxidant properties of the acetone, methanol and water extracts of A. arctotoides root extracts were screened through ABTS (2, 2'-azino-bis (3-ethylbenzothiazoline-6-sulphonic acid), FRAP (ferric reducing ability of plasma) and DPPH (1,1-diphenyl-2-picrylhydrazyl) radical scavenging effects (Afolayan et al., 2007). For the DPPH assay, the methanol, water and acetone extracts showed a lower potency than ascorbic acid and BHT at all tested concentrations. In the ABTS assay, the scavenging activity of the acetone root extract (96.7\%) was not significantly higher than that of BHT (96.3\%) at $0.1 \mathrm{mg} / \mathrm{ml}$ and similarly, the FRAP values for the acetone (80.43) and methanol (81.86) extracts were comparable to that of BHT (62.3).

\section{Toxicity}

The toxicity tests undertaken on A. arctotoides reported in the literature are summarized in Table 2 . Three toxicity screens for the crude extracts of $A$. arctotoides on cell lines, rats and brine shrimp were identified in the literature. Graded doses of the aqueous extract from the shoot of $A$. arctotoides $(400,800,1,600$ and $3,200 \mathrm{mg} / \mathrm{kg}$ body weight) were separately administered to mice in a test group while the control group was treated with orally administered distilled water $(3 \mathrm{ml} / \mathrm{kg}$ ) (Jimoh et al., 2008). Clinical chemistry and histopathological examination of major organs were performed on all the animals. The extract did not cause any significant change in the red blood cell count, packed cell volume, haemoglobin concentration, mean corpuscular volume, mean corpuscular haemoglobin concentration, and mean corpuscular haemoglobin even up to the dose of $2000 \mathrm{mg} / \mathrm{kg}$ body weight. No significant lesions were observed in the organs examined and no behavioral changes or deaths were recorded in the mice after $48 \mathrm{hr}$ of administration of the extract. The extract also caused significant decrease in the level of platelets, total conjugated and un-conjugated bilirubin, blood urea nitrogen (BUN) in all the experimental groups when compared to the control group (Jimoh et al., 2008). However, all doses caused a significant increase in the levels of white blood cell count and its differentials. Based on the findings of Jimoh et al. (2008), it may be inferred that the aqueous extract of $A$. arctotoides has no toxic effect on the red blood cell parameters. The decrease in the level of platelets induced by the extracts indicates that the continued administration of the extract may lead to haemorrhage, however, considering the absence of neither mortality nor behavioral changes in the treated animals (Jimoh et al., 2008), the plant may be safe for medicinal uses.

Otang et al., (2013a) investigated the cytotoxicity of the hexane and acetone extracts of Arctotis arctotoides against the Chang Liver cell line using the in vitro MTT assay. The $\mathrm{IC}_{50}$ values for the acetone and hexane extracts were 17.4 and $12.4 \mu \mathrm{g} / \mathrm{ml}$ respectively, lower than that of grieseofulvin $(9.02 \mu \mathrm{g} / \mathrm{ml})$, which was used as positive control. In another study (Otang et al., 2013b), the hexane and acetone extracts of $A$. arctotoides were assayed for toxicity to hatching and larval mortality of Artemia salina. Based on Meyer's toxicity index, the acetone extract of A. arctotoides with $\mathrm{LC}_{50}$ values $>1 \mathrm{mg} / \mathrm{ml}$ was considered as non-toxic and may be further explored for the development of plant-based pharmaceuticals (Otang et al., 2013b).

\section{Conclusion}

A. arctotoides is an important medicinal plant in South Africa and continues to be frequently prescribed in traditional medicine especially by the rural people of Eastern Cape Province for the treatment of epilepsy, indigestion and catarrh of the stomach, skin infections and wounds (van der Walt, 2002). Justification of its traditional use has been supported by a number of studies detailing the bioactivity of the crude extracts and the isolated phytochemicals (Tables $1 \& 2$ ). Crude extracts and phytochemicals from the root and shoot of $A$. arctotoides were reported to have demonstrated potent anti-microbial properties against a number of bacteria and fungi. Three of the compounds isolated from the shoot of $A$. arctotoides (Sultana et al., 2008) showed moderate antibacterial activity. In addition, the hexane extract of the plant showed high antifungal activity with a broad spectrum of action. These results provide partial evidence regarding the traditional use of A. arctotoides in the treatment of wounds in Eastern Cape of South Africa.

One of the traditional uses of A. arctotoides is the management of skin diseases (Otang et al., 2014). However, no studies have been carried out on the anti-inflammatory properties of A. arctotoides extract although inflammation is common to many skin problems. Skin diseases have been of major concern recently due to the association of skin opportunistic infections and HIV/AIDS. With an HIV prevalence rate of $16.6 \%$ amongst South Africans aged between 15 - 49 years, South Africa has the largest HIV epidemic in the world (Statistics South Africa, 2011). Skin diseases such as dermatitis, prurigo, scabies, and papular urticaria are either untreated or over-treated with strong topical steroids or antibiotics which have been found to cause considerable disability (Njoroge and Bussmann, 2007). These short-comings, including the appearance of drug-resistant microbial strains have resulted in increased efforts for the search of better antimicrobial agents and much attention is now being directed towards natural products (Abbasi et al., 2010). Hence, further research on the anti-inflammatory properties of A. arctotoides seems warranted.

Given the widespread search for alternative medicines with novel mechanisms of actions from plants, it is surprising that there are currently no reports on the traditional uses of A. arctotoides outside the borders of South Africa. This endemism of A. arctotoides to South Africa may partially account for the limited amount of scientific research that has been carried out on the plant. Hence, in order to fully exploit the medicinal potential of $A$. arctotoides, there are perhaps two main areas for potential development. The first is to ensure the expansion of existing traditional knowledge from the areas where A. arctotoides is currently used for traditional medicine into neighboring communities where the plant is not used for the stated indications. Ultimately such knowledge expansion and sharing will support the greater use of the plant in primary healthcare (TRAMIL, 2011). Secondly, the possible mechanisms explaining the antibacterial and antifungal activity deserve to be investigated. 
http://dx.doi.org/10.4314/ajtcam.v11i6.12

\section{Acknowledgments}

We greatly appreciate the Govan Mbeki Research and Development Centre (GMRDC) of the University of Fort Hare and the National Research Foundation (NRF), South Africa for funding this project.

\section{References}

1. Abbasi, A.M., Khan, M.A., Ahmad, M., Zafar, M., Jahan, S., and Sultana, S. (2010). Ethnopharmacological Application of Medicinal Plants to Cure Skin Diseases and in Folk Cosmetics among the Tribal Communities of North-West Frontier Province, Pakistan. J. Ethnopharmacol. 128: 322-335.

2. Afolayan, A.J. (2003). Extracts from the Shoots of Arctotis arctotoides inhibit the Growth of Bacteria and Fungi. Pharm. Biol., 41(1): 22-25.

3. Afolayan, A.J., Jimoh, F.O., Sofidiya, M.O., Koduru, S. and Lewu, F.B. (2007). Medicinal Potential of the Root of Arctotis arctotoides. Pharm. Biol., 45: 486-493.

4. Afolayan, A.J., Grierson, D.S., and Otang, W.M. J. (2014). Ethnopharmacol. Ethnobotanical Survey of Medicinal Plants used in the Management of Skin Disorders among the Xhosa Communities of the Amathole District, Eastern Cape, South 153: 220-232.

5. Bremer, K. (1994). Asteraceae, Cladistics and Classification. Timber Press, Portland, Oregon.

6. Fouche, G., Cragg, G.M., Pillay, P., Kolesnikova, N., Maharaj, V.J. and Senabe, J. (2008). In vitro Anticancer Screening of South African Plants. J. Ethnopharmacol., 119:455-461.

7. Funk, V.A., Bayer, R.J., Keeley, S., Chan, R., Watson, L., Gemeinholzer, B., Schilling, E., Panero, J.L., Baldwin, B.G., Garcia- Jacas, N., Susanna, A., and Jansen, R.K. (2005). Everywhere but Antarctica: Using a Supertree to Understand the Diversity and Distribution of the Compositae. Biol. Skr., 55: $343-374$

8. Jeffrey, C. (2007). Compositae: Introduction with Key to Tribes. Families and Genera of Vascular Plants, 3: 61-87.

9. Jimoh, F. O., Adedapo, A. A., Sofidiya, M. O., Masika, P. J. and Afolayan, A. J. (2008). Safety Evaluation of the Extract from the Shoots of Arctotis arctotoides in Rats and Mice. Afr. J. Biotechnol., 7: 3173-3177.

10. Mckenzie, R.J., Herman, P.P.J. and Barker N.P. (2006). Arctotis decurrens (arctotideae), the Correct Name for A. merxmuelleri and A. scullyi. Bothalia., 36: 2.

11. Njoronge, G.N., Bussmann, R. W. (2007). Ethnotherapeutic Management of Skin Diseases among the Kikuyus of Central Kenya. J. Ethnopharmocol., 111: 303-307.

12. Otang, W.M, Grierson, D.S., and Ndip, R.N. (2012a). Ethnobotanical Survey of Medicinal Plants used in the Management of Opportunistic Fungal Infections in HIV/AIDS Patients in the Amathole District of the Eastern Cape Province, South Africa. J. Med. Plants Res. 6: 2071-2080.

13. Otang, W.M, Grierson, D.S., and Ndip, R.N. (2013a). Assessment of Potential Toxicity of three South African Medicinal Plants Using the Brine Shrimp (Artemia salina) Assay. Afr. J. Pharm. Pharmacol., 7: 1272-1279.

14. Otang, W.M., Grierson, D.S. and Ndip, R.N. (2013b). Cytotoxicity of three South African Medicinal Plants using the Chang Liver Cell Line. Afr. J. Tradit Complement Altern Med. 11: 324-329.

15. Otang, W.M., Grierson, D.S., Ndip, R.N. (2012b). Antifungal Activity of Arctotis arctotoides (L.f.) O. Hoffm. and Gasteria bicolor Haw. Against Opportunistic Fungi Associated with Human Immunodeficiency Virus/acquired Immunodeficiency Syndrome. Phcog. Mag. 8:135-140.

16. Oyedeji, O. A., Yani, V.V. and Afolayan, A.J. (2005). Chemical Composition of the Essential Oil from Arctotis arctotoides (L.F.) O. Hoffm. (syn. Vendium arctotoides Less.). Flavour Fragr. J., 2005; 20: 232-234.

17. Pope, G. V. (1992). Compositae. In G. V. Pope [ed.] Flora Zambesiaca: Mozambique, Malawi, Zambia, Zimbabwe and Botswana. 6: 1-264 Royal Botanic Gardens, Kew, England.

18. Statistics SouthAfrica. (2011). Mid Year Population Estimates. Accessed 3/01/2013. http://www.statssa.gov.za/Publications/statsdown load.asp?PPN=P0302.

19. Sultana, N and Afolayan, A.J. (2007). A novel daucosterol derivative and antibacterial activity of compounds from Arctotis arctotoides, Nat. Prod. Res.: Formerly Nat. Prod. Lett., 21: 889-896.

20. Sultana, N. and Afolayan, A.J. (2003). Bioactive Sesquiterpene Lactones Isolated from the Shoots of Arctotis arctotoides. S. Afr. J. Bot., 69: 1-3.

21. Sultana, N., Afolayan, A.J. and Bhuiyan, R.A. (2008). Antimicrobial Compounds from the Shoots of Arctotis Arctotoides. Bangladesh J. Sci. Ind. Res. 43: 89-96.

22. TRAMIL. (2011). Program of Applied Research for Traditional Popular Medicine in the Caribbean. from /http://www.tramil.net/english/Tramil.html.

23. Van der Walt, L. (2002). Arctotis arctotoides (L.f.) O.Hoffm. South African National Biodiversity Institute's Plant Information. Website www.plantzafrica.com. 